\title{
Comparison principle and stability for a class of stochastic fractional differential equations
}

\author{
Yuli Lu' ${ }^{1}$ Zhangsong Yao ${ }^{2}$, Quanxin Zhu ${ }^{1,3^{*}}$, Yi Yao $^{3}$ and Hongwei Zhou ${ }^{2}$
}

\footnotetext{
"Correspondence: zqx22@126.com 'Department of Mathematics, Ningbo University, Ningbo, Zhejiang 315211, China

${ }^{3}$ School of Mathematical Sciences, Institute of Finance and Statistics, Nanjing Normal University, Nanjing, Jiangsu 210023, China

Full list of author information is available at the end of the article
}

\begin{abstract}
In this paper, we study a class of stochastic fractional differential equations. We first establish a novel comparison principle for such equations. Then, we use the new comparison principle to obtain some stability criteria, which include the stability in probability, uniform stability in probability, asymptotic stability in probability, and pth moment exponential stability. Finally, an example is provided to illustrate the obtained results.
\end{abstract}

Keywords: comparison principle; stochastic fractional differential equation; stability in probability; uniform stability in probability; asymptotic stability in probability; pth moment exponential stability

\section{Introduction}

In recent decades, stochastic models have been applied in many areas such as social science, physical science, finance, control engineering, mechanical, electrical and industry. The stability analysis is one of the most important research topics in stochastic models. There has been a large number of stability results in the literature. For instance, see [1] and the references therein.

On the other hand, fractional calculus is a mathematical subject with a history of more than 300 years. There have been more and more researchers interested in studying the fractional calculus in the last twenty years. One of the main reasons is that the integerorder calculus and conventional differential equations are no longer suitable tools for many systems and processes, such as viscoelastic system [2], dielectric polarization [3], electrode-electrolyte polarization [4], electrical circuit [5], electromagnetic waves [6], heat condition [7], biological system [8], quantitative finance [9], and quantum evolution of complex system [10]. However, such systems can be elegantly described by fractionalorder differential equations with the help of the fractional calculus.

In comparison with the classical integer-order calculus, the fractional calculus has natural advantages in describing systems possessing memory and hereditary properties. In recent years, the classical mathematical modeling approaches coupled with the stochastic methods have been used to develop stochastic dynamic models for financial data (stock price). In order to extend this approach to more complex dynamic processes in sciences and engineering operating under internal structural and external environmental perturbations, we establish stochastic fractional differential equations by introducing the concept of dynamics processes operating under a set of linearly independent time-scales.

o2014 Lu et al.; licensee Springer. This is an Open Access article distributed under the terms of the Creative Commons Attribution License (http://creativecommons.org/licenses/by/2.0), which permits unrestricted use, distribution, and reproduction in any medium, provided the original work is properly cited. 
Recently, the authors in [11] studied the problem of existence and uniqueness of solutions of the initial value problem of stochastic fractional differential equations. But they did not discuss the stability analysis problem. This situation encourages our present research.

Motivated by the above discussion, in this paper we investigate the stability analysis problem for a class of stochastic fractional differential equations. Different from the traditional Lyapunov stability theory, we first establish a novel comparison principle for stochastic fractional differential equations, and then obtain some stability criteria including the stability in probability, uniform stability in probability, asymptotic stability in probability, $p$ th moment stability of such equations based on the new comparison principle. Finally, we use an example to illustrate our stability results.

The rest of this paper is organized as follows. In Section 2, we introduce the model of a class of stochastic fractional differential equations, some preliminary results and definitions. In Section 3, we construct the comparison principle for stochastic fractional differential equations of Itô-Doob type and obtain some stability criteria including the stability in probability, uniform stability in probability, asymptotic stability in probability, $p$ th moment stability of such equations. An example is provided to illustrate how to apply the developed results in the stability analysis in Section 4. Finally, in Section 5, we conclude the paper with some general remarks.

\section{Preliminary description and problem formulation}

Throughout this paper, unless otherwise specified, $\mathbb{R}$ denotes the set of real numbers, $\mathbb{R}_{+}$ denotes the set of positive real numbers, $Z$ denotes the set of integers and $N$ is the set of positive integers. Let $B(t)=\left(B_{1}(t), B_{2}(t), \ldots B_{m}(t)\right)$ be an $m$-dimensional Brownian motion defined on a complete probability space $(\Omega, \mathcal{F}, P)$, let $d^{\alpha} x$ denote the differential of order $\alpha$, and let $\|\cdot\|$ denote the Euclidean norm in $\mathbb{R}^{n}$.

Definition 1 (R-L fractional integral $[10,12])$ Let $f(t)$ be a continuous function defined on the interval $[a, b]$, where $a, b \in \mathbb{R}$ and $a<b$. Then, for $v \in(0,1)$, we define the RiemannLiouville fractional integral as follows:

$$
{ }_{a} D_{t}^{-v} f(t)=\frac{1}{\Gamma(v)} \int_{a}^{t}(t-\xi)^{v-1} f(\xi) d \xi
$$

where $\Gamma(\cdot)$ is the gamma function defined by

$$
\Gamma(z)=\int_{0}^{\infty} t^{z-1} e^{-t} d t
$$

Definition 2 (R-L fractional derivative [13]) Let $f(t) \in C[a, b], l \in \mathbb{R}_{+}, m \leq l<m+1$, and then the Riemann-Liouville derivative is defined as

$$
{ }_{a} D_{t}^{l} f(t)={ }_{a} D_{t}^{m+1}\left({ }_{a} D_{t}^{-v} f(t)\right), \quad v=m+1-l>0 .
$$

Submitting (1) into (2), we have

$$
{ }_{a} D_{t}^{l} f(t)=\frac{1}{\Gamma(-l+m+1)}\left(\frac{d}{d t}\right)^{m+1} \int_{a}^{t}(t-\xi)^{m-l} f(\xi) d \xi
$$


When $l$ is a nonnegative integer, then equality (3) represents the classical derivative of integer order. However, the properties of differential and integral with integer order are different. For instance, letting $f(t) \equiv c$ in equality (3), where $c$ is a constant, then we can obtain its $l$ th derivative,

$$
{ }_{a} D_{t}^{l} c=\frac{c(t-a)^{-l}}{\Gamma(-l+1)} \neq 0
$$

which is clearly different from the differential with integer order.

Definition 3 (Multi-time scale integral [11]) For $p \in N, p>1$, let $\left\{T_{1}, T_{2}, \ldots, T_{p}\right\}$ be a set of linearly independent time-scales. Let $f:[a, b] \times \mathbb{R}^{p-1} \rightarrow \mathbb{R}^{n}$ be a continuous function defined by $f(t):=f\left(T_{1}(t), T_{2}(t), \ldots, T_{p}(t)\right)$. The multi-time scale integral of the composite function $f$ over an interval $\left[t_{0}, t\right] \subseteq(a, b)$ is defined as the sum of $p$ integrals with respect to the time-scales $T_{1}, T_{2}, \ldots, T_{p}$. We denote it by $I f$,

$$
(I f)(t)=\int_{t_{0}}^{t} f(s) d s=\sum_{j=1}^{p}\left(I_{j} f\right)(t)
$$

where the sense of the integral

$$
\left(I_{j} f\right)(t)=\int_{t_{0}}^{t} f(s) d T_{j}(s)
$$

depends on the time-scale $T_{j}$ for each $j=1,2, \ldots, p$.

Definition 4 (Multi-time scale differential [11]) Let $f$ be a function defined in Definition 3. The multi-time scale differential of the composite function $f$ is defined to be the sum of the partial differentials of $f$ with respect to the times-scales $T_{1}(t), T_{2}(t), \ldots, T_{p}(t)$. We denote it by $d f$,

$$
(d f)(t)=\sum_{j=1}^{p}\left(d_{j} f\right)(t)
$$

where for each $j=1,2, \ldots, p$,

$$
\begin{aligned}
\left(d_{j} f\right)(t)= & f\left(T_{1}(t), \ldots, T_{j-1}(t), T_{j}(t+\Delta t), T_{j+1}(t), \ldots, T_{p}(t)\right) \\
& -f\left(T_{1}(t), \ldots, T_{j-1}(t), T_{j}(t), T_{j+1}(t), \ldots, T_{p}(t)\right),
\end{aligned}
$$

$\Delta t \simeq d t$ for small $\Delta t$, and $\left(d_{j} f\right)(t)$ corresponds to the integral $\left(I_{j} f\right)(t)$ in Definition 3. In particular, if the function $f$ has continuous partial derivatives with respect to each timescale, then the following holds:

$$
(d f)(t)=\sum_{j=1}^{p} \frac{\partial f}{\partial T_{j}}(t) d T_{j}(t) .
$$

Remark 1 For $p=3$, consider the linearly independent set consisting of time-scale $T_{1}(t)=$ $t$, which signifies the ideal and controlled environmental condition; $T_{2}(t)=B(t)$, where $B$ 
is an $m$-dimensional Brownian motion on a complete probability space $\Omega \equiv(\Omega, \mathcal{F}, P)$; and $T_{3}(t)=t^{\alpha}, 0<\alpha<1$ indicates the time-varying delay or lagged process. Under this set of time-scale, the following stochastic fractional differential equation of Itô-Doob type is suggested:

$$
d x=b(t, x) d t+\sigma_{1}(t, x) d B(t)+\sigma_{2}(t, x)(d t)^{\alpha}, \quad x\left(t_{0}\right)=x_{0},
$$

where $\alpha \in(0,1), b(t, x) \in C\left[\mathbb{R}_{+} \times \mathbb{R}^{n} ; \mathbb{R}^{n}\right], \sigma_{1}(t, x) \in C\left[\mathbb{R}_{+} \times \mathbb{R}^{n} ; \mathbb{R}^{n \times m}\right], \sigma_{2}(t, x) \in C\left[\mathbb{R}_{+} \times\right.$ $\left.\mathbb{R}^{n} ; \mathbb{R}^{n}\right]$

Remark 2 The differentials $d t, d B(t)$, and $(d t)^{\alpha}$ are in the sense of Cauchy-Riemann or Lebesgue [14], Itô-Doob [15], and Jumarie [16, 17], respectively.

Assume that $b, \sigma_{1}$, and $\sigma_{2}$ satisfy the Lipschitz condition and linear growth condition, and thus it follows from [11] that system (4) has a unique solution $x(t)$. Also, assume that $b(t, 0) \equiv 0, \sigma_{1}(t, 0) \equiv 0, \sigma_{2}(t, 0) \equiv 0$, and then system (4) admits a trivial solution or zero solution $x(t) \equiv 0$ corresponding to the initial data $x_{0}=0$.

Remark 3 We remark that some classical models are special cases of system (4).

(i) If $\sigma_{2}(\cdot, \cdot)=0$ in Remark 1, then (4) is reduced to the following Itô-Doob type stochastic differential equation:

$$
d x=b(t, x) d t+\sigma_{1}(t, x) d B(t), \quad x\left(t_{0}\right)=x_{0} .
$$

(ii) Letting $\sigma_{1}(\cdot, \cdot)=0$ in (4), then we have the following generalized version of the classical deterministic fractional differential equation:

$$
d x=b(t, x) d t+\sigma_{2}(t, x)(d t)^{\alpha}, \quad x\left(t_{0}\right)=x_{0} .
$$

(iii) If $b(\cdot, \cdot) \equiv 0$ and $\sigma_{1}(\cdot, \cdot) \equiv 0$, then (4) becomes the following deterministic fractional differential equation:

$$
D_{t_{0}}^{\alpha} x=\sigma_{2}(t, x), \quad x\left(t_{0}\right)=x_{0} .
$$

Take $S_{h} \doteq\{x \mid\|x\|<h\} \subset \mathbb{R}^{n}$, and then $S_{h}$ is an open set and $0 \in S_{h}$. Let $C\left[\mathbb{R}_{+} \times S_{h}, \mathbb{R}^{m}\right]$ denote the family of all nonnegative functions $V(t, x)$ on $\mathbb{R}_{+} \times S_{h}$, which are continuously twice differentiable in $x$ and differentiable in $t$. If $V \in C\left[\mathbb{R}_{+} \times S_{h}, \mathbb{R}^{m}\right]$, then by the Itô's formula and (4), we have the following:

$$
d V(t, x)=\mathcal{L}_{1} V(t, x) d t+\mathcal{L}_{2} V(t, x) d B(t)+\mathcal{L}_{3} V(t, x)(d t)^{\alpha}
$$

where

$$
\begin{aligned}
& \mathcal{L}_{1} V(t, x)=V_{t}(t, x)+V_{x}(t, x) b(t, x)+\frac{1}{2} \sigma_{1}^{T}(t, x) V_{x x}(t, x) \sigma_{1}(t, x), \\
& V_{t}(x, t)=\frac{\partial V(x, t)}{\partial t}, \quad V_{x}(x, t)=\left(\frac{\partial V(x, t)}{\partial x_{1}}, \ldots, \frac{\partial V(x, t)}{\partial x_{n}}\right),
\end{aligned}
$$




$$
\begin{aligned}
& V_{x x}(x, t)=\left(\frac{\partial^{2} V(x, t)}{\partial x_{i} \partial x_{j}}\right)_{n \times n}, \\
& \mathcal{L}_{2} V(t, x)=V_{x}(t, x) \sigma_{1}(t, x), \quad \mathcal{L}_{3} V(t, x)=V_{x}(t, x) \sigma_{2}(t, x) .
\end{aligned}
$$

Definition 5 (Lyapunov stable)

(i) The zero solution $x(t) \equiv 0$ of system (4) is said to be Lyapunov stable if for every $\varepsilon>0$ and $t_{0} \in[0, \infty)$, there exists $\delta=\delta\left(\varepsilon, t_{0}\right)>0$ such that $\left\|x\left(t, t_{0}, x_{0}\right)\right\|<\varepsilon$ for all $t>t_{0}$ when $\left\|x_{0}\right\|<\delta$.

(ii) The zero solution of system (4) is uniformly Lyapunov stable if for every $\varepsilon>0$, there exists $\delta=\delta(\varepsilon)>0$ such that $\left\|x\left(t, t_{0}, x_{0}\right)\right\|<\varepsilon$ for all $t>t_{0}$ when $\left\|x_{0}\right\|<\delta(\varepsilon)$.

(iii) The zero solution of system (4) is asymptotically stable if it is Lyapunov stable and there exists $\delta\left(t_{0}\right)>0$ such that $\lim _{t \rightarrow \infty} x(t)=0$ when $\left\|x_{0}\right\|<\delta\left(t_{0}\right)$.

Definition 6 (Stable in probability) The zero solution $x(t) \equiv 0$ of system (4) is said to be stable in probability if for every $\varepsilon_{1} \in(0,1)$ and $\varepsilon_{2}>0$, there exists $\delta=\delta\left(\varepsilon_{1}, \varepsilon_{2}, t_{0}\right)>0$ such that

$$
P\left\{\left\|x\left(t, t_{0}, x_{0}\right)\right\|<\varepsilon_{2}, t \geq t_{0}\right\} \geq 1-\varepsilon_{1},
$$

when $\left\|x_{0}\right\|<\delta$.

Definition 7 (Asymptotically stable in probability) The zero solution $x(t) \equiv 0$ of system (4) is asymptotically stable if it is stable in probability, and for every $\eta \in(0,1)$, there exists $\delta=\delta\left(\eta, t_{0}\right)>0$ such that

$$
P\left\{\lim _{t \rightarrow \infty} x\left(t, t_{0}, x_{0}\right)=0\right\} \geq 1-\eta
$$

when $\left\|x_{0}\right\|<\delta$.

Definition 8 ([18]) A function $\varphi(z)$ is said to belong to the class $\mathcal{K}$ if $\varphi \in C\left[\mathbb{R}_{+}, \mathbb{R}_{+}\right], \varphi(0)=$ 0 and $\varphi(z)$ is strictly increasing in $z$. A function $\varphi(z)$ is said to belong to the class $\mathcal{V} \mathcal{K}$ if $\varphi$ belongs to $\mathcal{K}$ and $\varphi$ is convex. A function $\varphi(t, z)$ is said to belong to the class $\mathcal{C K}$ if $\varphi \in C\left[\mathbb{R}_{+} \times \mathbb{R}_{+} ; \mathbb{R}_{+}\right], \varphi(t, 0)=0$, and $\varphi(t, z)$ is concave and strictly increasing in $z$ for each $t \in \mathbb{R}_{+}$.

Lemma $1([19,20])$ Let $f(t)$ be a continuous function, then the solution of the following equation:

$$
d x=f(t)(d t)^{\alpha}, \quad t \geq 0, \quad x(0)=x_{0}, \quad 0<\alpha \leq 1
$$

is defined by the equality

$$
\int_{0}^{t} f(\tau)(d \tau)^{\alpha}=\alpha \int_{0}^{t}(t-\tau)^{\alpha-1} f(\tau) d \tau, \quad 0<\alpha \leq 1
$$

\section{Comparison principle and stability for stochastic fractional differential equations}

In this section, we present our main results. First of all, we give the comparison principle, which plays an important role in the proof of our results. 
Lemma 2 Assume that the following conditions are satisfied.

(i) $\left[t_{0}, T\right)(T \leq \infty)$ is the largest interval of existence of the maximal solution $u(t) \equiv u\left(t, t_{0}, u_{0}\right)$ of the following deterministic fractional differential equation:

$$
d u(t)=f(t, u(t)) d t+\varphi(t, u(t))(d t)^{\alpha}, \quad u\left(t_{0}\right)=u_{0},
$$

where $f, \varphi \in C\left[\left[t_{0}, T\right) \times \mathbb{R}^{n} ; \mathbb{R}^{n}\right]$ and $f(t, u), \varphi(t, u)$ are monotonically non-increasing in $u$ for each $t$, and $f(t, 0) \equiv 0, \varphi(t, 0) \equiv 0$.

(ii) $V \in C\left[\mathbb{R}_{+} \times \mathbb{R}^{n} ; \mathbb{R}_{+}\right]$, and for $(t, x) \in \mathbb{R}_{+} \times \mathbb{R}^{n}, \tau \in\left(t_{0}, t\right)$

$$
\mathbf{E} \mathcal{L}_{1} V(t, x(t)) \leq f(t, \mathbf{E} V(t, x(t)))+\alpha \varphi(t, \mathbf{E} V(t, x(t)))(t-\tau)^{\alpha-1}
$$

where $\mathcal{L}_{1} V$ is the operator defined in Section 2.

(iii) For the solution $x(t) \equiv x\left(t, t_{0}, x_{0}\right)$ of $(4), \mathbf{E} V(t, x(t))$ exists for $t \geq t_{0}$.

If $\mathbf{E}\left[V\left(t_{0}, x_{0}\right)\right] \leq u_{0}$, then

$$
\mathbf{E}[V(t, x(t))] \leq u\left(t, t_{0}, u_{0}\right)
$$

Proof We shall prove Lemma 2 by contradiction. Now suppose that (10) is not true, then there exists a constant $a>t_{0}$ such that

$$
\mathbf{E}[V(a, x(a))]>u\left(a, t_{0}, u_{0}\right)
$$

Since $\mathbf{E}\left[V\left(t_{0}, x_{0}\right)\right] \leq u_{0}$, by the continuity of $u(t)$ and $\mathbf{E}[V(t, x(t))]$, we see that there exists a constant $b \in\left(t_{0}, a\right)$ satisfying

$$
\mathbf{E}[V(b, x(b))]=u(b)
$$

Noting that $f(t, u)$ and $\varphi(t, u)$ are monotonically non-increasing in $u$ for all $t$, it follows from (9) and (11) that for each $s \in[b, a]$,

$$
\begin{aligned}
\mathbf{E} \mathcal{L}_{1} V(s, x(s)) & \leq f(s, \mathbf{E} V(s, x(s)))+\alpha \varphi(s, \mathbf{E} V(s, x(s)))(s-\tau)^{\alpha-1} \\
& \leq f(s, u(s))+\alpha \varphi(s, u(s))(s-\tau)^{\alpha-1} \\
& =\frac{d u(s)}{d s}
\end{aligned}
$$

Integrating both sides of the above inequality, we obtain

$$
\int_{b}^{a} \mathbf{E} \mathcal{L}_{1} V(s, x(s)) d s \leq \int_{b}^{a} \frac{d u(s)}{d s} d s=u(a)-u(b) .
$$

Thus, by using the Dynkin formula, we get

$$
\begin{aligned}
\mathbf{E}[V(a, x(a))]-\mathbf{E}[V(b, x(b))] & =\int_{b}^{a} \mathbf{E} \mathcal{L}_{1} V(s, x(s)) d s \\
& \leq \int_{b}^{a} \frac{d u(s)}{d s} d s \\
& =u(a)-u(b) .
\end{aligned}
$$


Recalling that $\mathbf{E}[V(b, x(b))]=u(b)$, the above inequality yields

$$
\mathbf{E}[V(a, x(a))] \leq u(a)
$$

which contradicts (11). Hence, (10) is satisfied. This completes the proof of Lemma 2.

As an application of the comparison principle, we will deduce some stability criteria for system (4).

Theorem 1 Assume that there exists a function $V(t, x) \in C\left[\mathbb{R}_{+} \times \mathbb{R}^{n} ; \mathbb{R}^{n}\right]$ such that the following two conditions are satisfied:

(1) $V(t, \cdot)$ is a locally Lipschitz continuous in $x$ and uniformly in $t$ compact set of $[0, \infty)$ satisfying

$$
\begin{aligned}
\mathbf{E}\left[\mathcal{L}_{1} V(t, x(t))\right] \leq & f(t, \mathbf{E} V(t, x(t))) \\
& +\alpha \varphi(t, \mathbf{E} V(t, x(t)))(t-\tau)^{\alpha-1}, \quad \forall(t, x) \in \mathbb{R}_{+} \times \mathbb{R}^{n},
\end{aligned}
$$

where $f$ and $\varphi$ are from Lemma 2.

(2) For every $(t, x) \in \mathbb{R}_{+} \times \mathbb{R}^{n}, V(t, x)$ satisfies

$$
\varphi_{1}(\|x\|) \leq V(t, x(t)) \leq \varphi_{2}(\|x\|)
$$

where $\varphi_{1}, \varphi_{2} \in \mathcal{K}$.

If the zero solution of (8) is Lyapunov stable, then the zero solution of (4) is stable in probability. Moreover, if the zero solution of (8) is uniformly stable, then the zero solution of (4) is uniformly stable in probability.

Proof Let $x(t)$ be the solution of (4), then by (12) we have

$$
\mathbf{E}\left[\varphi_{1}(\|x\|)\right] \leq \mathbf{E}[V(t, x(t))] .
$$

Now suppose that the zero solution of (8) is Lyapunov stable. Then it follows from the definition of Lyapunov stability that for any $0<\eta<1$ and $\varepsilon>0$, there exists $\delta_{1}=\delta_{1}\left(\varepsilon, \eta, t_{0}\right)>$ 0 such that if $u_{0}<\delta_{1}$, then $u\left(t, t_{0}, u_{0}\right) \leq \eta \varphi_{1}(\varepsilon), t \geq t_{0}$. Obviously, the function $\mathbf{E}[V(t, x(t))]$ is continuous with respect to $x$ since $V(t, x)$ is continuous with respect to $x$. Choosing $u_{0}=$ $V\left(t_{0}, x_{0}\right) \geq 0$, then for $\delta_{1}=\delta_{1}\left(\varepsilon, \eta, t_{0}\right)>0$, there exists $\delta_{2}=\delta_{2}\left(\delta_{1}\right)>0$ such that $\mathbf{E}\left[V\left(t_{0}, x_{0}\right)\right]=$ $\mathbf{E}\left[u_{0}\right]=u_{0}<\delta_{1}\left(\varepsilon, \eta, t_{0}\right)$ when $\left\|x_{0}\right\|<\delta_{2}$. So it follows from Lemma 2 that

$$
\mathbf{E}[V(t, x(t))] \leq u\left(t, t_{0}, u_{0}\right) \leq \eta \varphi_{1}(\varepsilon) .
$$

By using the Chebyshev inequality and (13)-(14), we have

$$
\begin{aligned}
P[\|x(t)\| \geq \varepsilon] & =P\left[\varphi_{1}(\|x(t)\|) \geq \varphi_{1}(\varepsilon)\right] \\
& \leq \frac{1}{\varphi_{1}(\varepsilon)} \mathbf{E}\left[\varphi_{1}(\|x(t)\|)\right] \\
& \leq \frac{1}{\varphi_{1}(\varepsilon)} \mathbf{E}[V(t, x(t))] \\
& \leq \frac{\eta \varphi_{1}(\varepsilon)}{\varphi_{1}(\varepsilon)}=\eta,
\end{aligned}
$$


and so

$$
P\left[\|x(t)\| \leq \varepsilon, \forall t \geq t_{0}\right] \geq 1-\eta .
$$

Therefore, from the definition of the stability in probability, we see that the zero solution of (4) is stable in probability. Furthermore, we suppose that the zero solution of (8) is uniformly stable. Noting that the constants $\delta_{1}, \delta_{2}$ in the above proof are independent of $t_{0}$, we can prove similarly that $\delta$ does not depend on $t_{0}$, which verifies that the zero solution of (4) is uniformly stable in probability. The proof of Theorem 1 is completed.

Theorem 2 Assume that all the conditions of Theorem 1 are satisfied. If the zero solution of (8) is asymptotically stable, then the zero solution of (4) is asymptotically stable.

Proof Suppose that the zero solution of (8) is asymptotically stable. Then, for any $\eta \in(0,1)$ and $\varepsilon>0$, there exists a positive constant $\delta_{0}=\delta_{0}\left(\eta, t_{0}\right)>0$ such that

$$
u(t)<\eta \varphi_{1}(\varepsilon), \quad t \rightarrow \infty,
$$

when $u_{0}<\delta\left(t_{0}\right)$. Choosing $u_{0}=V\left(t_{0}, x_{0}\right) \geq 0$, then by Theorem 1 , inequality (12) and the continuity of $\mathbf{E}[V(t, x(t))]$, we obtain

$$
\begin{aligned}
& \mathbf{E}[V(t, x(t))] \leq u(t)<\eta \varphi_{1}(\varepsilon), \quad t \rightarrow \infty, \\
& P\left\{\left\|x\left(t, t_{0}, x_{0}\right)\right\|<\varepsilon, t \rightarrow \infty\right\} \geq 1-\eta .
\end{aligned}
$$

Hence, there exists $\delta_{0}=\delta_{0}\left(\eta, t_{0}\right)>0$ such that

$$
P\left\{\lim _{t \rightarrow \infty} x\left(t, t_{0}, x_{0}\right)=0\right\} \geq 1-\eta,
$$

when $\left\|x_{0}\right\|<\delta_{0}$. This together with the definition of asymptotic stability in probability implies that the zero solution of (4) is asymptotically stable in probability. This completes the proof of Theorem 2 .

Theorem 3 Assume that all the conditions of Theorem 1 are satisfied. Moreover, for any $p \geq 1$,

$$
\varphi_{1}\left(\|x(t)\|^{p}\right) \leq V(t, x(t)) \leq \varphi_{2}\left(\|x(t)\|^{p}\right), \quad \forall(t, x) \in \mathbb{R}_{+} \times \mathbb{R}^{n},
$$

where $\varphi_{1} \in \mathcal{V} \mathcal{K}, \varphi_{2} \in \mathcal{C K}$. If the zero solution of (8) is Lyapunov stable, then the zero solution of (4) is pth moment exponentially stable.

Proof By using Jensen's inequality and (15), we obtain

$$
\begin{aligned}
0 & \leq \varphi_{1}\left(\mathbf{E}\left[\|x(t)\|^{p}\right]\right) \leq \mathbf{E}\left[\varphi_{1}\left(\|x(t)\|^{p}\right)\right] \leq \mathbf{E}[V(t, x(t))] \\
& \leq \mathbf{E}\left[\varphi_{2}\left(\|x(t)\|^{p}\right)\right] \leq \varphi_{2}\left(\mathbf{E}\left[\|x(t)\|^{p}\right]\right) .
\end{aligned}
$$


For the solution $x(t)=x\left(t, t_{0}, x_{0}\right)$ of $(4)$, it follows from Lemma 2 that

$$
\mathbf{E}[V(t, x(t))] \leq u\left(t, t_{0}, u_{0}\right)
$$

when $\mathbf{E}\left[V\left(t_{0}, x_{0}\right)\right] \leq u_{0}$.

Now suppose that the zero solution of (8) is Lyapunov stable. Then, for any $\varepsilon>0$ and $\varphi_{1}(\varepsilon)>0$, there exists $\delta_{1}=\delta_{1}\left(t_{0}, \varepsilon\right)$ such that

$$
u\left(t, t_{0}, u_{0}\right) \leq \varphi_{1}(\varepsilon), \quad t \geq t_{0}
$$

when $u_{0} \leq \delta_{1}$.

Let us choose $x_{0}$ such that $u_{0}=\varphi_{2}\left(\mathbf{E}\left[\left\|x_{0}\right\|^{p}\right]\right)$ and $\mathbf{E}\left[V\left(t_{0}, x_{0}\right)\right] \leq u_{0}$. Recalling that $\varphi_{2} \in$ $\mathcal{C K}$, there exists $\delta=\delta(\varepsilon)$ such that $u_{0}=\varphi_{2}\left(\mathbf{E}\left[\left\|x_{0}\right\|^{p}\right]\right)<\delta$, when $\mathbf{E}\left[\left\|x_{0}\right\|^{p}\right]<\delta_{1}$. Hence, by (16)-(18), we obtain

$$
\varphi_{1}\left(\mathbf{E}\left[\|x(t)\|^{p}\right]\right) \leq \varphi_{1}(\varepsilon), \quad t \geq t_{0} .
$$

This fact together with $\varphi_{1} \in \mathcal{V} \mathcal{K}$ yields that

$$
\mathbf{E}\left[\|x(t)\|^{p}\right] \leq \varepsilon, \quad t \geq t_{0} .
$$

Therefore, from the definition of the $p$ th moment exponential stability, we see that the zero solution of (4) is $p$ th moment exponentially stable. The proof of Theorem 3 is completed.

\section{An example}

Consider the following stochastic fractional differential system:

$$
\left\{\begin{array}{l}
d x_{1}(t)=x_{2}(t) d t+\left(x_{1}(t)+x_{2}(t)\right)(d t)^{\alpha}, \\
d x_{2}(t)=\left(-2 x_{1}(t)-x_{2}(t)\right) d t+\left(\frac{1}{2} x_{1}(t)-x_{2}(t)\right) d B(t)+\left(x_{1}(t)-x_{2}(t)\right)(d t)^{\alpha},
\end{array}\right.
$$

where $\alpha \in(0,1), t \in[0, \infty)$.

Letting $V(t, x(t))=2 x_{1}(t)^{2}+x_{1}(t) x_{2}(t)+x_{2}(t)^{2}$, and then we have

$$
\begin{aligned}
V(t, x(t)) & \geq 2 x_{1}(t)^{2}-\frac{1}{2} x_{1}(t)^{2}-\frac{1}{2} x_{2}(t)^{2}+x_{2}(t)^{2} \\
& \geq \frac{1}{2}\left[x_{1}(t)^{2}+x_{2}(t)^{2}\right] \\
& =\frac{1}{2}\|x(t)\|^{2} \\
V(t, x(t)) & \leq 2 x_{1}(t)^{2}+\frac{x_{1}(t)^{2}+x_{2}(t)^{2}}{2}+x_{2}(t)^{2} \\
& \leq \frac{5}{2}\left[x_{1}(t)^{2}+x_{2}(t)^{2}\right] \\
& =\frac{5}{2}\|x(t)\|^{2} .
\end{aligned}
$$


Obviously, $V(t, x(t))$ is locally Lipschitz continuous in $x$ and uniformly in $t$,

$$
\begin{aligned}
\mathbf{E} \mathcal{L}_{1} V(t, x(t))= & {\left[4 x_{1}(t)+x_{2}(t)\right] x_{2}(t)+\left[x_{1}(t)+2 x_{2}(t)\right]\left[-2 x_{1}(t)-x_{2}(t)\right] } \\
& +\left[\frac{1}{2} x_{1}(t)-x_{2}(t)\right]\left[\frac{1}{2} x_{1}(t)+x_{2}(t)\right] \\
= & -\frac{7}{4} x_{1}(t)^{2}-x_{1}(t) x_{2}(t)-2 x_{2}(t)^{2} \\
\leq & -x_{1}(t)^{2}-\frac{1}{2} x_{1}(t) x_{2}(t)-\frac{1}{2} x_{2}(t)^{2} \\
\leq & -\frac{1}{2} V(t, x(t))+\alpha V(t, x(t))(t-\tau)^{\alpha-1},
\end{aligned}
$$

where $\tau \in(0, t)$. Thus, for the stochastic fractional differential system (19), the comparison function can be chosen as

$$
d u(t)=-\frac{1}{2} u(t) d t+u(t)(d t)^{\alpha}, \quad u(0)=u_{0} .
$$

The solution of equation (20) is

$$
u(t)=u(0) E_{\alpha}\left[\frac{\alpha}{\alpha-1} \Gamma(1+\alpha) t^{\alpha-1}\right] e^{-\frac{1}{2} t},
$$

where $E_{\alpha}(x)$ denotes the Mittag-Leffler function

$$
E_{\alpha}(x)=\sum_{k=0}^{\infty} \frac{x^{k}}{\Gamma(1+\alpha k)} .
$$

For more details about the Mittag-Leffler function, we refer the reader to [16]. It is obvious that the solution of (20) is stable. So, according to Theorem 1, the zero solution of stochastic fractional differential equation (19) is stable in probability.

\section{Conclusion}

In this paper, we have established a novel comparison principle for a class of stochastic fractional differential systems. By employing the new comparison principle and Lyapunov stability theory, we obtain some useful stability criteria. These criteria are drawn from the stability of the comparison function with regard to the original system and an inequality constraint condition. As an application, an example is presented to illustrate how to apply the developed results in the stability analysis. The example shows that the proposed method is very convenient.

Competing interests

The authors declare that they have no competing interests.

Authors' contributions

All authors contributed equally to the writing of this paper. All authors read and approved the final manuscript.

\section{Author details}

'Department of Mathematics, Ningbo University, Ningbo, Zhejiang 315211, China. ${ }^{2}$ School of Mathematics and Information Technology, Nanjing Xiaozhuang University, Nanjing, Jiangsu 211171, China. ${ }^{3}$ School of Mathematical Sciences, Institute of Finance and Statistics, Nanjing Normal University, Nanjing, Jiangsu 210023, China. 


\section{Acknowledgements}

This work was jointly supported by the National Natural Science Foundation of China (61374080), the Natural Science Foundation of Zhejiang Province (LY12F03010), the Natural Science Foundation of Ningbo (2012A610032), K.C. Wong Magna Fund in Ningbo University, and a Project Funded by the Priority Academic Program Development of Jiangsu Higher Education Institutions.

Received: 3 February 2014 Accepted: 17 July 2014 Published: 15 Aug 2014

\section{References}

1. Mao, X: Stochastic Differential Equation and Application. Horwood, Chichester (1997)

2. Bagley, RL, Calico, RA: Fractional order state equations for the control of viscoelastic structures. J. Guid. Control Dyn. 14(2), 304-311 (1991)

3. Sun, HH, Abdelwahad, AA, Onaral, B: Linear approximation of transfer function with a pole of fractional order. IEEE Trans. Autom. Control 29(5), 441-444 (1984)

4. Ichise, M, Nagayanagi, Y, Kojima, T: An analog simulation of non-integer order transfer functions for analysis of electrode process. J. Electroanal. Chem. Interfacial Electrochem. 33(2), 253-256 (1971)

5. Chen, G, Friedman, EG: An RLC interconnect model based on Fourier analysis. IEEE Trans. Comput.-Aided Des. Integr. Circuits Syst. 24(2), 170-183 (2005)

6. Heaviside, O: Electromagnetic Theory. Chelsea, New York (1971)

7. Jenson, VG, Jeffreys, GV: Mathematical Methods in Chemical Engineering, 2nd edn. Academic Press, New York (1997)

8. Anastasio, TJ: The fractional-order dynamics of brainstem vestibule-oculumotor neurons. Biol. Cybern. 72(1), 69-79 (1994)

9. Laskin, N: Fractional market dynamics. Physica A 287, 482-492 (2000)

10. Kusnezov, D, Bulgac, A, Dang, GD: Quantum Lévy processes and fractional kinetics. Phys. Rev. Lett. 82(6), 1136-1139 (1999)

11. Pedjeu, JC, Ladde, GS: Modeling, method and analysis. Chaos Solitons Fractals 45(3), 279-293 (2012)

12. Kilbas, AA, Srivastava, HM, Trujillo, JJ: Theory and Applications of Fractional Differential Equations. Elsevier, Amsterdam (2006)

13. Ladas, GE, Lakshmikantham, V: Differential Equations in Abstract Spaces. Academic Press, New York (1972)

14. Lang, S: Real and Functional Analysis, 3rd edn. Springer, New York (1993)

15. Gihman, II, Skorohod, AV: Stochastic Differential Equations. Springer, New York (1972)

16. Jumarie, G: Fractional Brownian motions via random walk in the complex plane and via fractional derivative. Comparison and further results on their Fokker-Planck equations. Chaos Solitons Fractals 22(4), 907-925 (2004)

17. Jumarie, G: New stochastic fractional models for Malthusian growth, the Poissonian birth process and optimal management of populations. Math. Comput. Model. 44(3-4), 231-254 (2006)

18. Dong, F, Wu, Y, Fang, Y: Comparison principle and stability of general continuous time Markov jump system. In: Proceedings of the 2008 International Conference on Computational Intelligence and Security, vol. 2, pp. 186-191 (2008)

19. Jumarie, G: On the representation of fractional Brownian motion as an integral with respect to $(\mathrm{d} t)^{a}$. Appl. Math. Lett. 18(7), 739-748 (2005)

20. Jumarie, G: Modified Riemann-Liouville derivative and fractional Taylor series of nondifferentiable functions further results. Comput. Math. Appl. 51(9-10), 1367-1376 (2006)

10.1186/1687-1847-2014-221

Cite this article as: Lu et al.: Comparison principle and stability for a class of stochastic fractional differential

equations. Advances in Difference Equations 2014, 2014:221

\section{Submit your manuscript to a SpringerOpen ${ }^{\circ}$ journal and benefit from:}

- Convenient online submission

- Rigorous peer review

Immediate publication on acceptance

- Open access: articles freely available online

- High visibility within the field

- Retaining the copyright to your article 